\title{
Theranostic Nanoplatform to Target Macrophages Enables the Inhibition of Atherosclerosis Progression and Fluorescence Imaging of Plaque
}

\section{Qi Wang}

Xuzhou Medical University

\section{Yong Wang}

Xuzhou Medical University

Siwen Liu

Xuzhou Medical University

Xuan Sha

Xuzhou Medical University

Xiaoxi Song

Xuzhou Medical University

Yue Dai

Xuzhou Medical University

Mingming Zhao

Xuzhou Medical University

Lulu Cai

Xuzhou Medical University

Kai Xu

The Affiliated Hospital of Xuzhou Medical University: Xuzhou Medical University Affiliated Hospital Jingjing Li ( $\sim$ qingchao0124@163.com )

Xuzhou Medical University https://orcid.org/0000-0002-6149-3350

\section{Research}

Keywords: Atherosclerosis, Theranostic nanomedicines, Macrophage, Fluorescence imaging

Posted Date: May 27th, 2021

DOl: https://doi.org/10.21203/rs.3.rs-533800/v1

License: (c) (1) This work is licensed under a Creative Commons Attribution 4.0 International License.

Read Full License 
Version of Record: A version of this preprint was published at Journal of Nanobiotechnology on July 28th, 2021. See the published version at https://doi.org/10.1186/s12951-021-00962-w. 


\section{Abstract}

Background: Rupture of atherosclerotic plaque can cause acute malignant heart and cerebrovascular events, such as acute coronary heart disease, stroke and so on, which seriously threaten the safety of human life and property. Therefore, the early diagnosis and inhibition of atherosclerotic plaque progress still be a vital task.

Results: In this study, we presented the development of composite mesoporous silica nanoparticle (CMSN) - based nanomedicines (NMs) (Ru(bpy) ${ }_{3} @ S_{2} \mathrm{O}_{2}-\mathrm{mSiO}_{2} @ S R T 1720 @$ AntiCD36,

CMSN@SRT@Anti) for accurate diagnosis and treatment of atherosclerosis. In vitro cell experiments showed that both RAW264.7 and ox-LDL-stimulated RAW264.7 cells could significantly uptake CMSN@SRT@Anti. Conversely, little fluorescence signal could be observed in CMSN@SRT group, showing the excellent targeting ability of CMSN@SRT@Anti to CD36 on macrophage. Additionally, such fluorescence signal was significantly stronger in ox-LDL-stimulated RAW264.7 cells, showing the ox-LDL can promote the upregulation of CD36 expression on macrophages to produce a stronger binding signal. For another, compared with free SRT1720,CMSN@SRT@Anti had a better and more significant effect on the inhibition of macrophage foaming process, which indicated that drug-carrying mesoporous silicon with targeting ability could enhance the efficacy of SRT1720. Animal experimental results showed that after the abdominal injection of CMSN@SRT@Anti, the aortic lesions of ApoE-/-mice could be observed with obvious and persistent fluorescence signals. After 4 weeks post- treatment, the serum total cholesterol, aortic plaque status and area were significantly improved in the mouse, and the effect was better than that in the free SRT1720 group or the CMSN@SRT group.

Conclusions: The designed CMSN@SRT@Anti with excellent biocompatibility, high-performance and superior atherosclerosis-targeting ability has great potential for accurate identification and targeted therapy of atherosclerotic diseases.

\section{Background}

Cardiovascular disease (CVD) is a widely recognized human killer, and as a representative CVD, coronary heart disease (CHD) has a very high mortality rate, accounting for more than half of the deaths of CVD cases worldwide every year [1, 2]. Atherosclerosis (AS) is the basic lesion of CVD. With the progression of AS, the rupture of gradually enlarged plaque can lead to vascular stenosis or occlusion, causing CHD and stroke [3]. Therefore, the accurate identification and effective intervention of AS is of great significance in preventing the occurrence of life-threatening events and disabilities.

The pathological process of AS is complex and involves many pathological mechanisms. In essence, it is a process of progressive aggravation of chronic inflammation, which is characterized by the deposition of lipids and various inflammatory cells under arterial intima $[4,5]$. Ox-LDL is an important participant in the occurrence and development of AS. Macrophages phagocyte ox-LDL through scavenger receptors and then transform into foam cells [6]. Based on this mechanism, some diagnosis and therapy complex 
have been fabricated for AS. IK17 (the first human single chain FV antibody fragment) was reported to be able to bind with the lipid and protein regions of ox-LDL and inhibit the phagocytosis of ox-LDL by macrophages. Shaw Px et al. injected iodinated IK17 (125I-IK17) into AS mice and realized the precise therapy for AS with ox-LDL as the target [7]. Except ox-LDL, a series targets have also been chosen for the specific diagnosis and therapy of AS. Macrophages can mediate the circulation of inflammatory cells in AS lesions and promote the formation of AS plaques by summoning fibroblasts. Thus, macrophage as the target was employed to diagnosis and treatment of AS. The transporter protein (TSPO) is significantly expressed on the surface of macrophages, and the TSPO ligand iodine 125-DPA-713 prepared by Foss CA et al. can efficiently accumulate in AS lesions, thus achieving the molecular diagnosis and treatment of AS with macrophages as the target [8]. Because the proliferation and migration of vascular smooth muscle cells (VSMCs) present in AS lesions is related to the formation of fibrous cap, profilin-1 highly expressed on the surface of VSMCs was also chosen as the target [9]. Zhang SH et al. covalently combined PFN1 (Profilin-1 Antibody) with paramagnetic $\mathrm{Fe}_{3} \mathrm{O}_{4}$ to obtain near-infrared fluorescence imaging/MRI nanoprobe, which could locate AS plaques monitoring by imaging. In addition, they could also moderate the progression of atherosclerosis [10]. In the early stage of AS, neutrophils appear in the lesions of AS, which was used as molecular targets for the ultra-early diagnosis of AS. Elastic cell enzyme exists in neutrophils plasmids and can be employed as target to indicate the presence of neutrophils. Glinzer A et al. prepared a near-infrared imaging agent Neutrophil Elastase 680 FAST to target elastic cell enzyme to realize the early diagnosis of AS [11]. In the late stage of AS, fibrin deposits on the plaque surface. Therefore, fibrin as a molecular target was introduced for the diagnosis of advanced AS. Replacing the DE loop of Simian virus 40 (SV40) major capsid protein VP1 with CREKA peptide which can target fibrin, Sun X et al. fabricated trifunctional SV40-based nanoparticles (VNPS) with QD800 encapsulation. Near-infrared fluorescence imaging shows that AS plaques at the late stage in live intact ApoE (-/-) could be noninvasively targeted and fluorescently imaged [12].

The above successful examples for the molecular imaging of AS plaque stimulates us to explore new probe to improve the theranostic functions. In this study, we selected Class B scavenger receptor (SR-B2) CD36 as a target for the molecular diagnosis and treatment of AS. Studies have shown that CD36, which is expressed on the surface of macrophage, is a key regulatory point for the formation and development of AS. It can mediate the conversion of macrophage into foam cell after phagocytosis of ox-LDL $[13,14]$. AntiCD36 can specifically bind to CD36 and inhibit macrophage phagocytosis of ox-LDL [15]. In order to strengthen the treatment effect on AS, SRT1720 was also introduced. As a specific activator of SIRT1, SRT1720 is 1000 times more active than resveratrol [16]. SRT1720 can mediate vasodilation, downregulate the expression of pro-inflammatory cytokines, promote the reverse transport of cholesterol in macrophages, inhibit macrophage foaminess, inhibit the expression of matrix metalloproteinases (MMPs), and prevent thrombosis and stabilize plaques [17-19]. Fluorescent dye, $\mathrm{Ru}(\mathrm{bpy})_{3} \mathrm{Cl}_{2}$ doped mesoporous silica, $\mathrm{Ru}(\mathrm{bpy})_{3} @ \mathrm{SiO}_{2}-\mathrm{mSiO}_{2}$ (CMSN) were employed as the nanocarrier to load SRT1720 and conjugate with AntiCD36. The synthetic process of Ru(bpy) ${ }_{3} @ S i O_{2}-\mathrm{mSiO}_{2} @ S R T 1720 @ A n t i C D 36$ (CMSN@SRT@Anti) was illustrated in Scheme 1. With the fluorescence imaging of $\mathrm{Ru}(\mathrm{bpy})_{3} \mathrm{Cl}_{2}$ and the 
targeted binding ability of AntiCD36 to CD36 overexpressed on the surface of macrophage, we could track the AS plaque and the inhibition effect of AS progress specifically both in vitro and in vivo.

\section{Results}

\section{Structure and characteristics of NMs}

The morphology of prepared $\mathrm{Ru}(\mathrm{bpy})_{3} @ \mathrm{SiO}_{2}$ and $\mathrm{CMSN}$ were observed by TEM. As shown in Fig. 1, spherical structure for $\mathrm{Ru}(\mathrm{bpy})_{3} @ \mathrm{SiO}_{2}$ with an average size of $28.57 \pm 2.53 \mathrm{~nm}$ and mesoporous structure for CMSN with an average size of $61.35 \pm 7.87 \mathrm{~nm}$ were displayed with well dispersion. Fourier transform infrared spectra (FT-IR), UV-vis absorption spectra and zeta potential determination were employed to monitor the whole assembly procedure of CMSN@Anti (Fig. 2a-c).FT-IR showed that CMSN@Anti had the $-\mathrm{CH}$ of AntiCD36 at the peak of $2958.65 \mathrm{~cm}^{-1}$ and $2923.93 \mathrm{~cm}^{-1}$. UV-vis absorption spectra displayed that the CMSN@Anti increased the characteristic absorption peak of AntiCD36 at 259 nm. Such a blue shift of absorption peak of AntiCD36 $(277 \mathrm{~nm})$ might be caused by the change of the force between adjacent particles after the binding of nanoparticles to Antibody [20]. The zeta potential of CMSN was $28.07 \pm 0.5 \mathrm{mV}$ and was changed to $24.47 \pm 0.4 \mathrm{mV}$ after amidation, as well as $8.63 \pm 0.3 \mathrm{mV}$ after further AntiCD36 conjugation. All of these characterizations indicated that AntiCD36 had been successfully attached to the surface of CMSN.

To be further used as a fluorescent agent, their fluorescence property was evaluated (Fig. 2d-f). The results of photobleaching resistance of CMSN under $365 \mathrm{~nm}$ UV lamp for different time showed that only a $15 \%$ loss of CMSN after $60 \mathrm{~min} 365 \mathrm{~nm}$ UV exposure. At room temperature, CMSN still maintained a strong fluorescence signal after $96 \mathrm{~h}$ under natural light. In addition, the fluorescence signal of CMSN was relatively stable at different $\mathrm{pH}$ values from 1 to 10 , and no obvious fluorescence quenching phenomenon was observed. It should be mentioned that compared with $\mathrm{Ru}(\mathrm{byp})_{3} \mathrm{Cl}_{2}, \mathrm{CMSN}$ presented better fluorescence stability under $365 \mathrm{~nm}$ UV exposure (Additional file 1: Fig. S1a). On the other hand, the hydrodynamic diameter of CMSN@Anti changed litter after storage of 9 days at room temperature, showing their excellent stability and potentials for biomedical applications. The average hydrodynamic diameters were determined to be $225.3 \mathrm{~nm}, 231.6 \mathrm{~nm}, 267.8 \mathrm{~nm}$ and $255.4 \mathrm{~nm}$ at the first, second, fifth and ninth day (Additional file 1: Fig. S1b). The excitation and emission spectrum of CMSN was shown in Fig. $2 \mathrm{~g}$ and its fluorescence quantum yield was determined to be 0.52 by Williams gradient method[21] with Rhodamine $6 \mathrm{G}$ as the reference (Fig. $2 \mathrm{~h}$ ).

Additional file 1: Fig. S2a,b shows the UV-vis absorption spectra of SRT1720 at different concentrations and their corresponding linear fitting graphs. According to the calculation, the encapsulation efficiency (EE) and loading efficiency (LE) of CMSN@SRT@Anti were determined to be $47 \pm 4 \%$ and $42 \pm 2 \%$ when the concentration of SRT1720 was 3 mM. The drug release curve of CMSN@SRT@Anti in different pH values of PBS is shown in Fig. 2i. The effect of drug release in neutral and weak acidic environment is stronger than that in alkaline environment. Under neutral or weak acid conditions, the SRT1720 release could reach $69 \pm 3 \%$ or $74 \pm 5 \%$ for 48 h, respectively. 


\section{Targeting ability of CMSN@SRT@Anti on macrophages, and its inhibition on macrophage foaming}

Macrophages, as the core cells of AS, are important components involved in the occurrence and development of AS. In this study, mouse macrophage cell line, RAW264.7 was used as the experimental object. Prior to study the targeted fluorescence imaging and macrophage foaming inhibition of CMSN@SRT@Anti, thiazole blue tetrazole bromination (MTT) assay was first introduced to evaluate their cytotoxicity. As shown in Additional file 1: Fig S3, the survival rate of RAW264.7 or NIH-3T3 cells were not significantly reduced even when the concentration of CMSN@SRT@Anti was $40 \mu \mathrm{g} / \mathrm{mL}$. One-way ANOVA was used to analyze the absorbance values of each treatment group $\left(F_{\mathrm{RAW} 264.7}=1.679, P=0.142>0.05\right.$; $\left.F_{\mathrm{NIH}-3 \mathrm{~T} 3}=1.737, P=0.128>0.05\right)$. Therefore, a concentration of CMSN@SRT@Anti below $40 \mu \mathrm{g} / \mathrm{mL}$ was chosen for the following cell imaging and the evaluation of inhibition effect of macrophage foaming.

The level of total cholesterol (TC) in cells could reflect the degree of lipid deposition to indicate the degree of cell foaming. Compared with the model group (MG), either CMSN, CMSN@Anti or AntiCD36 could reduce the cell TC value little (Fig. 3a-c: $P>0.05$ ). Figure 3d showed that CMSN@SRT at $16 \mu \mathrm{g} / \mathrm{mL}$ or 24 $\mu \mathrm{g} / \mathrm{mL}$ could decrease the cell TC value compared with MG (** $P<0.01)$, but there was no significant difference in the effect between the two groups $(P>0.05)$. But CMSN@SRT@Anti at $8 \mu \mathrm{g} / \mathrm{mL}, 16 \mu \mathrm{g} / \mathrm{mL}$, or $24 \mu \mathrm{g} / \mathrm{mL}$ could all reduce the cell TC value compared with MG and the effect was enhanced with the concentration increased (Fig. 3e). Free SRT1720 at $5 \mu \mathrm{g} / \mathrm{mL}$ or $7.5 \mu \mathrm{g} / \mathrm{mL}$ could decrease the cell TC value compared with MG $\left({ }^{* * P}<0.01\right)$, but there was no significant difference in the effect between the two groups $(P>0.05)$ (Fig. 3f). With the same SRT1720 concentration, CMSN@SRT@Anti displayed lower intracellular TC contents than free SRT1720 or CMSN@SRT, showing the best inhibition effect of macrophage forming was achieved with the help of AntiCD36 (Fig. 4a).

In order to clearly visually demonstrate these inhibitory effects of each group on macrophage foaming, the treated cells were measured by Oil Red $O$ staining. Similar with the results of TC analysis, the Oil Red 0-positive areas in RAW264.7 cells were enhanced after pre-treated with ox-LDL. But they were reduced after co-incubation with free SRT1720,CMSN@SRT or CMSN@SRT@Anti and reached the lowest point forCMSN@SRT@Anti treatment.CMSN,CMSN@Anti or AntiCD36 alone displayed no influence on the macrophage foaming (Fig. 4b,c).

The targeting ability of CMSN@SRT@Anti to macrophage was performed by fluorescence imaging. Both RAW264.7 and ox-LDL-stimulated RAW264.7 cells displayed a strong red fluorescent signal in cytoplasm after interacting with CMSN@SRT@Anti. Conversely, little fluorescence signal could be observed in CMSN@SRT group (Fig. 5a), showing the excellent targeting ability of CMSN@SRT@Anti to CD36 on macrophage. Additionally, such cytoplasmic fluorescence signal was significantly stronger for ox-LDLstimulated RAW264.7 cells (Fig. 5b: $* \star \star P<0.001$ vs. unstimulated cells), showing that the ox-LDL can promote the upregulation of CD36 expression on macrophages [14] to produce a stronger binding signal. Figure $5 \mathrm{c}$ shows that there was almost no fluorescent signal could be observed in NIH-3T3 cells. 
On the whole, our fabricated CMSN@SRT@Anti can target macrophages, especially activated macrophages stimulated by ox-LDL and enhance the efficacy of SRT1720 to effectively inhibit the foaming process of macrophages.

\section{In vivo toxicity evaluation and metabolism}

After intraperitoneal injection of CMSN@SRT@Anti to Kunming mice for 1d, 1w or 3w, the blood test results (additional file 1: Fig. S4) showed that compared with the control group, there was no significant difference in the chosen blood indicators (Alanine aminotransferase (ALT), Total protein (TP), albumin (ALB), Creatinine (CREA), urea(UREA), White blood cells (WBC), Red blood cell (RBC), Hemoglobin (HGB), Hematocrit( $\mathrm{HCT})$, Mean Corpuscular Hemoglobin(MCH), Mean red blood cell volume (MCV)) $(P>0.05)$, except a transient increase in Aspartate aminotransferase (AST), which returned to normal level after $1 \mathrm{w}$. The body weight of the mice showed a slight weight loss on the first day after CMSN@SRT@Anti injection and a gradual increase thereafter, with a growth curve similar to that of the control group (additional file 1: Fig. S5a). Histological examinations of major organs of Kunming mice were performed intraperitoneal administration of CMSN@SRT@Anti for $1 \mathrm{~d}$ and 3 w. It showed that no organic lesions, inflammation or other abnormalities were observed in the liver, spleen, heart, kidney and lung of mice (additional file 1: Fig. S5b). To better understand the metabolism of the injected nanomaterials in mice, visceral fluorescence was detected at different time points post-injection. As shown in additional file 1: Fig. 6, the fluorescence emissions changes from weak-strong-weak in liver and cholecyst with the time passing, indicating the possible excreted route of CMSN@SRT@Anti was through the hepatobiliary systems. The fluorescence signal of all organs basically returned to normal level after $24 \mathrm{~h}$.

\section{Specific targeting and progress inhibition of AS plaque in mice}

In order to select the best imaging time point of nanoparticles in vivo, CMSN@SRT@Anti were intraperitoneally injected into AS model mice. The fluorescence imagings of aorta at different time points were collected and shown in Fig. 7a. The results displayed that continuous fluorescence signal was observed in the atherosclerotic lesions of mice after CMSN@SRT@Anti injection, and the strongest signal appeared at time points $1 \mathrm{~h}, 4 \mathrm{~h}$ and $8 \mathrm{~h}$, especially $4 \mathrm{~h}$. Therefore, $4 \mathrm{~h}$ was selected as the time point to compare the plaque targeting ability of CMSN@SRT@Anti, CMSN@SRT and PBS. As shown in Fig. 7b, the fluorescence signal in the aorta of mice treated with CMSN@SRT@Anti was significantly higher than that of CMSN@SRT or PBS ( ${ }^{*} * P<0.001$ vs. CMSN@SRT or PBS).

In order to visually display the anti-atherosclerosis effect of CMSN@SRT@Anti, the aortas of mice with the intervention were dissected and stained with Oil Red $\mathrm{O}$ and the ORO-positive area were quantitatively analyzed (Fig. 8a).This image showed that with the same SRT1720 concentration, CMSN@SRT@Anti, CMSN@SRT or free SRT1720 could all reduce the aorta red staining area of ApoE-/- mice compared with Vehicle group (** $P<0.01)$. Notably, CMSN@SRT@Anti reduced the area of aorta red stain more effectively than CMSN@SRT (** $P<0.01$ ) or SRT1720 ( $\left.{ }^{*} P<0.05\right)$, indicating CMSN@SRT@Anti had the best anti-AS effect in vivo. We also carried out quantitative analysis on serum TC values of mice in each group after different treatments. Learnt from Fig. 8b, the injection of CMSN@SRT@Anti, CMSNs@SRT or free 
SRT1720 can all reduce the blood TC value of AS model mice ( $* \star P<0.01$ vs. Vehicle group). Similarly, CMSN@SRT@Anti treatment resulted in a significant decrease in serum TC content than CMSN@SRT or SRT1720 $(* * P<0.01)$

Histological cross-sections were stained with H\&E to further confirm such inhibition effect (Fig. 8c). Compared with the Vehicle group, the proportion of AS plaque area and lumen cross section in mice treated with CMSN@SRT@Anti was significantly reduced. In addition, H\&E staining on the main viscera of mice treated with CMSN@SRT@Anti showed that no significant organic lesions, inflammation or other abnormalities were observed in the major organs of ApoE-/-mice after 4 weeks post-injection of CMSN@SRT@Anti, displaying their excellent biocompatibility (Fig. 8d).

\section{Discussion}

At present, theranostic nanomedicines (NMs) related technology is becoming mature and they have been widely used in the scientific research of various diseases, such as cancer, metabolism-related diseases, immune-related diseases, etc., and many cancer- related NMs have completed clinical transformation [22]. By comparison, NMs for atherosclerosis (AS) is rare. To construct a nanoprobe to target AS plaque and deliver drug for the inhibition of $A S$ progress, $\mathrm{Ru}(\mathrm{bpy}){ }_{3} \mathrm{Cl}_{2}$ doped mesoporous silicon was chosen as the nanocarrier in this work. Mesoporous silicon (MSN) has been widely used in molecular diagnosis and treatment of various diseases and has been recognized as a General Recognized as Safe (GRAS) by the U.S. Food and Drug Administration (FDA) for its non-toxic, stable and biocompatible properties. MSN can prevent drug crystallization and improve drug solubility through nanoscale partition. Hollow MSN is an ideal drug-loading nanoplatform with low density, large specific surface area, strong surface plasticity and large drug loading capacity [23-25]. On the other hand, fluorescence imaging photosensitizer $\mathrm{Ru}(\mathrm{bpy})_{3} \mathrm{Cl}_{2}$ can emit orange-red fluorescence under UV lamp (365 nm reflective light source), and it is widely used in fluorescence labeling due to its good photobleaching resistance and wide detection range. Specifically, when $\mathrm{Ru}(\mathrm{bpy})_{3} \mathrm{Cl}_{2}$ was sealed in silicon spheres, its fluorescence stability can be significantly improved [26], which has also been testified in this work. Thus, based on the selective etching method [27], $\mathrm{Ru}(\mathrm{bpy})_{3} @ \mathrm{SiO}_{2}-\mathrm{mSiO}_{2}(\mathrm{CMSN})$ as a platform was fabricated to load drug and conjugate with targeting molecule for the diagnosis and treatment of AS.

In nitrogen-protected anhydrous toluene, the surface amination of CMSN was achieved after the reaction of CMSN with appropriate amount of APTES for only $5 \mathrm{~h}$. After amination, the solubility of $\mathrm{CMSN}-\mathrm{NH}_{2}$ was further improved, and the surface charge was changed from $-28.07 \pm 0.5 \mathrm{mV}$ to $24.47 \pm 0.4 \mathrm{mV}$, which was consistent with the characterization of $\mathrm{mSiO}_{2}$ amination by Dai Y. et al. [28], indicating that $\mathrm{CMSN}-\mathrm{NH}_{2}$ was successfully prepared. Using rhodamine $6 \mathrm{G}$ as the reference material, the measured fluorescence quantum yield is as high as $52 \%$, indicating their promising potential for use as fluorescent signal reporter. Fluorescent substances with fluorescence quantum yields within the range of $10-100 \%$ are valuable for analysis and application, such as the commonly used fluorescent dyes rhodamine B and quinine sulfate, whose fluorescence quantum yields are $31 \%$ and $58 \%$ [21], respectively. Furthermore, their 
anti-photobleaching ability was also evaluated under the irradiation of $365 \mathrm{~nm}$ UV lamp for $1 \mathrm{~h}$. The experimental result showed that the fluorescence intensity decreased slightly with the extension of irradiation time, but the fluorescence signal still remained at a high level, indicating their good fluorescence stability. Under the condition of strong acid or strong base, the photosensitizer particles can agglomerate due to the decrease of repulsion force, thus promoting the fluorescence quenching of photosensitizer [29]. But in this work, $\mathrm{Ru}(\mathrm{bpy})_{3} \mathrm{Cl}_{2}$ was sealed in the silicon sphere, which could effectively block $\mathrm{Ru}(\mathrm{bpy})_{3} \mathrm{Cl}_{2}$ agglomeration. CMSN displayed a stable fluorescence signal under a wide range of $\mathrm{pH}$ (1-10) environments, which was consistent with the description of $\mathrm{Ru}(\mathrm{bpy})_{3} @ \mathrm{SiO}_{2}$ fluorescence stability by Ho Ta et al. [26]. After nine days of particle size stability test, the hydrated particle size of CMSN@Anti showed no significant change, indicating their excellent colloid stability, which was necessary for the further biomedical applications.

In recent years, a large number of studies have shown that macrophages play a decisive role in the occurrence, development and outcome of AS. Early ideas suggested that unstable plaques were characterized by a large necrotic center with a large infiltration of macrophages and a decrease in extracellular matrix content. A high proportion of macrophages was considered to be the main characteristic of vulnerable atherosclerotic plaques, VASPs [30,31]. The latest research results indicate that whether macrophages can promote the formation of VASPs mainly depends on the polarization state of macrophages rather than the number of macrophages. Under different cytokine environment, macrophages can differentiate into opposite subtypes, namely, pro-inflammatory macrophages (M1) and anti-inflammatory macrophages (M2). M1 macrophages are widely present in unstable plaques and can promote microcalcification deposition in the necrotic center of AS plaques through vesicle-mediated mineralization. M2 macrophages have the function of repairing and stabilizing plaques, and can promote the deposition of large calcifications by inducing the maturation of vascular smooth muscle cells (VSMCs) [32,33]. Therefore, the localization of M1-type macrophages has become the key point in the diagnosis and treatment of AS. Class II scavenger receptor CD36, expressed on the surface of macrophages, can mediate the uptake of ox-LDL by macrophages, and is a key factor in the polarization of macrophages to M1. It was reported that CD36 inhibitor SSO could significantly inhibit the polarization of macrophages to $M 1$ and make them transform into $M 2$ macrophages $[34,35]$. In a recent study by Iryna Voloshyna et al., CD36 was also found to be highly expressed on the surface of M1 macrophages, and the immunosuppressant cyclosporine ( $\mathrm{CsA}$ ) could significantly down-regulate the expression of CD36 on the surface of M1 macrophages [36]. Therefore, in this work, AntiCD36 was modified on the surface of $\mathrm{CMSN}-\mathrm{NH}_{2}$ to target $\mathrm{CD} 36$ for the track and treatment of AS plaque. The fabricated CMSN@Anti was analyzed by Zeta potential apparatus, UV-vis and FT-IR. UV-vis spectrum showed that AntiCD36 had a characteristic absorption peak at $277 \mathrm{~nm}$ compared with $\mathrm{CMSN}-\mathrm{NH}_{2}$, a characteristic absorption peak of the antibody at 259 nm emerged for CMSN@Anti, indicating that AntiCD36 was successfully connected to the surface of CMSN. The blue shift of AntiCD36's absorption peak from 277 $\mathrm{nm}$ to $259 \mathrm{~nm}$ after modification on CMSN might be caused by a change in the force between the adjacent particles after the antibody binding to the nanoparticle [20]. FT-IR spectra showed that the Si-OSi antisymmetric stretching vibration peak $\left(1076.22 \mathrm{~cm}^{-1}\right)$ of $\mathrm{mSiO}_{2}$ was observed in both CMSN@Anti 
and CMSN-NH${ }_{2}$, showing that the connection of AntiCD36 did not affect the basic structure of CMSN$\mathrm{NH}_{2}$. Compared with CMSN-NH ${ }_{2},-\mathrm{CH}$ peak of AntiCD36 at $2958.65 \mathrm{~cm}^{-1}$ and $2923.93 \mathrm{~cm}^{-1}$ was observed for CMSN@Anti. The zeta potential changes further proved the successful preparation of CMSN@Anti. The surface charges of CMSN-NH 2 , AntiCD36 and CMSN@Anti were determined to be $24.47 \pm 0.4 \mathrm{mV}$, $-12.83 \pm 2.39 \mathrm{mV}$ and $8.63 \pm 0.3 \mathrm{mV}$, respectively.

The existence of specific targeting molecule AntiCD36 not only favors the track of AS plaque, but also help to improve the drug delivery efficiency and therapy effect, eventually. Nicotinamide adenine dinucleotide (NAD+) dependent class III histone deacetylase (SIRT1) has been dubbed as the "longevity gene" [37], which can effectively resist aging, various tumors, neurodegeneration, cardiovascular diseases, and metabolic diseases. The increased expression of SIRT1 can effectively treat AS. Resveratrol, a traditional SIRT1 activator, has positive effects on cardiovascular, nervous system and cancer. However, the mechanism of its application in the treatment of AS is still unclear, which makes it difficult to predict the occurrence of adverse reactions in clinical application. In addition, recent studies have shown that besides SIRT1, resveratrol also has several other targets, such as PDES and PI3K. [38, 39]. SRT1720, as a SIRT1-specific activator, has been shown to be 1000 times more active than resveratrol [16]. In addition, due to its small molecular weight, SRT1720 is easy to be loaded into nanoparticles, which enables its application to be extended to the field of molecular medicine and further strengthens its therapeutic advantage for AS [38]. In this study, SRT1720 was loaded into the mesopore of CMSN for the in vitro and in vivo treatment of AS. With a 3 mM SRT1720, CMSN@SRT@Anti presented higher LE (42 $\pm 2 \%)$ and EE (47 $\pm 4 \%)$. The release effect of CMSN@SRT@Anti in neutral or weak acidic environment was obviously stronger than that in alkaline. Under alkaline environment, CMSN@SRT@Anti reached the release platform at $10 \mathrm{~h}$, and the release amount at $48 \mathrm{~h}$ was $35 \pm 2 \%$. But in weak acid environment, the release platform period was extended to $28 \mathrm{~h}$, and the release amount at $48 \mathrm{~h}$ could reach $74 \pm 5 \%$, which might come from the good solubility of SRT1720 under weak acid conditions. Similar with chronic inflammation in nature, AS lesions have acidic microenvironment [40]. Nanomaterials with high drug release efficiency in acidic environment have greater therapeutic potential. .

Studies have shown that the expression of SIRT1 is very low in the lesion during the development stage of AS, and the application of SIRT1 activator can stimulate the expression of SIRT1, thereby inhibiting the progression of AS and making it turn positive [41]. In this study, SRT1720 was loaded into the mesopore to explore its potential in the treatment of AS. We demonstrated the anti-AS superiority of CMSN@SRT@Anti in vitro and in vivo experiments. In vitro experiment results showed that CMSN@SRT@Anti could effectively reduce TC content in RAW264.7 cells. In addition, the TC lowering effect of CMSN@SRT@Anti was stronger than that of SRT1720 alone, indicating that CMSN@SRT@Anti with targeting ability can enhance the therapeutic effect of SRT1720 on AS [38]. Similar results were obtained in vivo. These findings not only confirm the anti-AS effect of SRT1720, but also expand the drug delivery method of SRT1720 through nanotechnology to enhance its therapy efficacy. It is worth noting that there was no statistical difference in the anti-AS effect between CMSN@SRT and SRT1720 in vitro. However, the effect of SRT1720 was better than CMSN@SRT in vivo. This might be caused by the 
different environment between in vitro and in vivo. In vitro, the relatively static culture environment in the cell dish was conducive to the phagocytosis of macrophages, while in vivo, the nanomaterials were constantly flowing in the blood circulation, and the accumulation of CMSN@SRT without targeting ability were less in the lesions of AS, which was not conducive to the treatment of AS. In addition, due to the low dose of AntiCD36 contained in CMSN@SRT@Anti, the NMs failed to show the competitive inhibitory effect of AntiCD36 on the treatment of AS [15].

The metabolism of NMs in vivo is an important index to evaluate the safety of NMs. The NMs that cannot be discharged from the body will be deposited in the body or cause damage to the body. Studies have shown that the distribution and metabolism of NMs in organisms are related to the size of NMs and the characteristics of viscera. NMs with particle size less than $5.5 \mathrm{~nm}$ are usually metabolized through the urinary system, while NMs with particle size greater than $15 \mathrm{~nm}$ are not easy to pass through the glomerulus and are eventually excreted to the cholecyst through the liver and enter the feces with bile [42]. The particle size of NMs synthesized in this study was larger than $15 \mathrm{~nm}$. The results of fluorescence imaging in mice suggested that CMSN@Anti was excreted by liver and cholecyst. Fluorescence signals appeared in the liver half an hour post drug injection, and began to appear in the cholecyst at 1 hour. Signals in the liver and cholecyst decreased to normal level at 24 hours, indicating that the NMs had been basically expelled, which was in line with the characteristics of large-particle size NMs metabolism in vivo. During cell imaging, with or without ox-LDL stimulation, compared with CMSN@SRT, the uptake of CMSN@SRT@Anti by macrophages was significantly increased, indicating the targeted ability of AntiCD36 to RAW264.7 cells, which was consistent with the relevant description in the literature $[13,14]$. In addition, compared with the untreated RAW264.7 cells, the uptake of CMSN@SRT@Anti in RAW264.7 cells after ox-LDL stimulation was significantly increased. This experimental phenomenon not only confirms the theory that ox-LDL can promote the upregulation of CD36 expression on macrophage surface [14], but also demonstrates that the NMs in this study have a better targeting effect on the lesion of acute advanced atherosclerosis. For in vivo fluorescence imaging, a continuous fluorescence signal was observed in the atherosclerosis lesion of mice after CMSN@SRT@Anti injection, and the signal could last at least for 24 h post-injection. Furthermore, the targeting ability of CMSN@SRT@Anti to AS lesions was significantly higher than that of CMSN@SRT or PBS. The results of mouse aorta imaging showed that the presence of AntiCD36 favored the location of CMSN@SRT@Anti at the AS lesions. Furthermore, the ApoE-/- mice were in good mental state during the 4 weeks of the NMs treatment, and no significant abnormalities were observed in organ pathology after treatment.

However, there are some limitations in this study. For example, the excitation visible fluorescent dye selected in this project has poor penetration, and its penetration in vivo is far less strong than that of near-infrared (NIR) fluorescent dye, resulting in an increase in experimental consumables and labor costs for NMS imaging and metabolism detection in vivo.

\section{Conclusion}


In the current study, we used mesoporous silicon as a nanoplatform to synthesize NMs for the precise diagnosis and treatment of atherosclerotic lesions. AntiCD36 was chosen as target molecule to recognize macrophages and SRT1720 loaded in mesoporous acted as the drug to inhibit the progress of AS plaque. It should be mentioned that such fabrication of functional NMs not only realized the tracking of AS lesions, but also enhanced the therapy efficacy of free SRT1720, which provided a new avenue for the applications of nanomaterials in the diagnosis and therapy of AS.

\section{Methods}

\section{Synthesis and characterization of CMSN@SRT@Anti}

The fabrication of the CMSN@SRT@Anti began with the preparation of Ru(bpy $)_{3} @ S_{2} \mathrm{O}_{2}$. Typically, $3.5 \mathrm{~mL}$ of $\mathrm{Ru}(\mathrm{bpy})_{3} \mathrm{Cl}_{2}$ solution ( $10 \mathrm{mM}$ in $\mathrm{H}_{2} \mathrm{O}$ ) was added to a mixture of $35 \mathrm{~mL}$ cyclohexane, TritonX-100 and hexanol with a ratio of 4:1:1. The obtained mixture was maintained at room temperature for 30 min under magnetic stirring. Then, $1 \mathrm{~mL}$ mixed solution of TEOS and $\mathrm{NH}_{3} \cdot \mathrm{H}_{2} \mathrm{O}$ with a ratio of $1: 1$ was added and was continued stirred for $24 \mathrm{~h}$. At the end of the reaction, the mixed solution was centrifuged (6000 rpm, $30 \mathrm{~min}$ ) to obtain a light red precipitate, namely $\mathrm{Ru}(\mathrm{bpy})_{3} @ \mathrm{SiO}_{2}$. The product was washed with ethanol for 3 times, and then dried in a lyophilizer and stored at $4^{\circ} \mathrm{C}$ away from light.

The covering of $\mathrm{mSiO}_{2}$ layer was used for drug delivery in the current study. Briefly, $25 \mathrm{~mL} \mathrm{~N}$ Hexadecyltrimethylammonium chloride (CTAC) aqueous solution (25\% wt) and $250 \mu \mathrm{L}$ triethylamine (TEA) were added into $35 \mathrm{~mL} \mathrm{Ru(bpy)})_{3} @ \mathrm{SiO}_{2}$ solution $(3 \mathrm{mg} / \mathrm{mL}$ ). After mixing evenly, $20 \mathrm{~mL}$ tetraethyl orthosilicate (TEOS) $(5 \%, \mathrm{v} / \mathrm{v})$ was added. The mixture was stirred at $60^{\circ} \mathrm{C}(200 \mathrm{rpm})$ for $24 \mathrm{~h}$ without light. After the reaction, the obtained product was demoulded by $\mathrm{NaCl}$-methanol solution (1\% wt) (3 times, $24 \mathrm{~h}$ each time). The obtained $\mathrm{Ru}(\mathrm{bpy})_{3} @ \mathrm{SiO}_{2}-\mathrm{mSiO}_{2}(\mathrm{CMSN})$ was washed, dried and finally preserved at $4^{\circ} \mathrm{C}$ in isolation from sunlight.

The CMSN@SRT@Anti was formed in three steps. Firstly, the amination of CMSN was performed. $20 \mu \mathrm{L}$ (3-Aminopropyl) triethoxysilane (APTES) was dropped in $3 \mathrm{~mL} \mathrm{CMSN}$ solution $(10 \mathrm{mg} / \mathrm{mL}$ in anhydrous toluene) slowly, and they were stirred at $60^{\circ} \mathrm{C}$ for $5 \mathrm{~h}$ under nitrogen protection. The precipitate was washed by anhydrous ethanol for three times and dried for later use. Secondly, SRT1720 was loaded. Briefly, $30 \mu \mathrm{L}$ SRT1720 solution ( $50 \mathrm{mM}$ in Dimethyl sulfoxide) were incubated with $470 \mu \mathrm{L}$ amino modified CMSN $(1.0 \mathrm{mg} / \mathrm{mL})$ overnight in a thermostatic oscillator $\left(37^{\circ} \mathrm{C}, 100 \mathrm{rpm}\right)$. After the reaction, the centrifugal precipitate was washed with PBS for three times to remove the unloaded drugs. Finally, covalent binding of CMSN@SRT with AntiCD36 was performed. $4 \mu \mathrm{g}$ AntiCD36 was dissolved in 1-(3Dimethylaminopropyl)-3-ethylcarbodiimide hydrochloride (EDC) solution (2 mg/mL in $500 \mu \mathrm{L}$ PBS) for $15 \mathrm{~min}$ for carboxyl group activation. Then, it was mixed with $500 \mu \mathrm{L}$ solution containing $1 \mathrm{mg}$ CMSN@SRT and $1 \mathrm{mg}$ N-hydroxysuccinimide (NHS) for $2 \mathrm{~h}$ incubation at $37^{\circ} \mathrm{C}$. After centrifugation and washing, the precipitates were dispersed in $1 \mathrm{~mL}$ PBS and stored at $4^{\circ} \mathrm{C}$ before use. The CMSN@Anti were prepared following the same procedures as described above, except without SRT loading. 
$\mathrm{Ru}(\mathrm{bpy})_{3} @ \mathrm{SiO}_{2}$ and CMSN were characterized by TEM (TECNAI- G2, FEl, USA). The absorption spectra and the fluorescence spectra were recorded by an ultra-micro spectrophotometer (NanoDrop One, Thermo Fisher Scientific, Waltham mass, USA ) and a fluorescence spectrophotometer (LS-55, PerkinElmer, USA), respectively. Infrared spectra were detected by Fourier transform infrared spectrometer (GB/T 2186 2007, Piketech, UK). The surface potential and hydrated particle size were detected by Zeta potentiometer (Nano ZS90, Malven, UK).

\section{Drug release in vitro}

In order to understand the drug loading and drug release of CMSN@SRT@Anti, the standard curve of SRT1720 was firstly determined with the absorbance intensity of SRT1720 at $246 \mathrm{~nm}$ as the ordinate and the corresponding concentrations $(0.01-5 \mathrm{mM})$ as the abscissa. The determinations of EE, LE and the drug release were all based on this.

The method of in vitro release is as follows. $0.5 \mathrm{~mL}$ CMSN@SRT@Anti $(0.25 \mathrm{mg} / \mathrm{mL})$ was transferred into a dialysis bag $(\mathrm{MWCO}=100 \mathrm{KDa})$ and placed in tubes containing PBS with $\mathrm{pH}$ of $6.5,7.4$, or 9 , respectively. They were rotated at $37^{\circ} \mathrm{C}$ with a speed of $200 \mathrm{rpm}$ for $48 \mathrm{~h} .2 \mu \mathrm{L}$ liquid in the tubes was picked up at scheduled time points, and their UV-vis absorbance values were measured using an ultramicro spectrophotometer. The PBS buffer volume in the test tube remains constant

\section{Cell Culture}

RAW264.7 (mouse macrophage cell line) and NIH-3T3 (mouse embryonic fibroblast cell line) were purchased from the Cell Bank of the Chinese Academy of Sciences (Shanghai, China). They were incubated with DMEM (Gibco, Logan, Utah, USA) supplemented with $10 \%$ fetal bovine serum (Thermo Fisher Scientific, Waltham mass, USA) and 1\% Penicillin-Streptomycin (Biyuntian, Shanghai, China). The cells were maintained in a $5 \% \mathrm{CO}_{2}$ incubator at $37^{\circ} \mathrm{C}$. For in vitro AS simulation, RAW264.7 cell were pretreated with $60 \mu \mathrm{g} / \mathrm{mL}$ ox-LDL (Yuanye Biotechnology, Shanghai, China).

\section{Biocompatibility evaluation in vitro and in vivo}

The cytotoxic effect of CMSN@SRT@Anti on RAW264.7 or NIH-3T3 was evaluated by the MTT method. After the incubation of RAW264.7 or NIH-3T3 with different concentrations of nanomaterials in 96-well plates $(\mathrm{n}=6)$ for $24 \mathrm{~h}(0-40 \mu \mathrm{g} / \mathrm{mL})$, the media was removed. After washing with PBS, $100 \mu \mathrm{L} 1 \mathrm{mg} / \mathrm{mL}$ MTT (Haoran Biotechnology, Shanghai, China) was added to each well. After further culture for $4 \mathrm{~h}$, the MTT solution was discarded gently and $100 \mu \mathrm{L}$ of Dimethyl sulfoxide (DMSO) (Pierce, Rockford, USA) was added to dissolve the formazan crystals. Finally, the absorbance of each well at $490 \mathrm{~nm}$ was measured with a microplate analyzer (Multiskon MK3, Thermo Fisher Scientific, Waltham mass, USA).

For in vivo biocompatibility evaluation, hematological and biochemical analyses of mouse with intraperitoneal injection of CMSN@SRT@Anti were carried out. The Kunming mice were treated with 
CMSN@SRT@Anti or an equivalent volume of PBS at a dose of $170 \mathrm{mg} / \mathrm{kg} / \mathrm{d}$ every other day. Eyeball blood samples were taken at 1 day, 7 day and 21 day after nanomaterials injection as well as 21 day after PBS injection. The collected blood was used for blood biochemical detection $(n=3)$ and routine blood detection $(n=3)$. Hematoxylin-eosin (H\&E) staining was performed for the main organs of the mice after different treatment and observed under an optical microscope.

To further study the metabolism of CMSN@SRT@Anti, the Kunming mice were injected intraperitoneally with the nanomaterials $(170 \mathrm{mg} / \mathrm{kg})$. The mice were sacrificed at scheduled time points $(0,0.5,1,2,4,6$, 12, 24h). The liver and cholecyst were collected and imaged (5200Multi, Ton, Shanghai, China).

\section{The evaluation of inhibition effect of macrophage cell foaming in vitro}

To further evaluate the therapy effect of CMSN@SRT@Anti in vitro, macrophage cells were treated with different groups: blank control group (BLK, RAW264.7 without any intervention), Model group (MG, RAW264.7 was incubated with ox-LDL only), CMSN group ( RAW264.7 was incubated with ox-LDL and CMSN), CMSN@Anti group (RAW264.7 was incubated with ox-LDL and CMSN@Anti),CMSN@SRT group (RAW264.7 was incubated with ox-LDL and CMSN@SRT), CMSN@SRT@Anti group (RAW264.7 was incubated with ox-LDL and CMSN@SRT@Anti), free AntiCD36 group (RAW264.7 was incubated with oxLDL and AntiCD36), and free SRT1720 group (RAW264.7 was incubated with ox-LDL and SRT1720). The concentrations of ox-LDL in all groups were $60 \mu \mathrm{g} / \mathrm{mL}$. After different treatments, the TC contents in cells were quantitatively analyzed using the TC assay kit (Suoqiao Biology, Shanghai, China) $(n=3)$.

In order to more intuitively demonstrate the inhibitory effect of different treatments on the RAW264.7 cell foaming, oil red staining was carried out to instead TC value determination. Image J software was used to semi-quantitatively analyze the red stained area $(n=3)$.

The cell oil red $O$ staining method was referred to the instruction. In brief, the treated cells were fixed with $4 \%$ paraformaldehyde (VICMED, Xuzhou, China) at room temperature for $15 \mathrm{~min}$. After rinsed with PBS twice, the oil red working solution was added to stain for 40 min in the dark. Then, the cells were rinsed with distilled water for three times and observed under a fluorescent inverted microscope. The configuration of the oil red 0 working liquid was prepared as follows. $6 \mathrm{~mL} 0.5 \%$ saturated oil red 0 was mixed with $4 \mathrm{~mL}$ distilled water, and purified by filtering through a filter paper in the dark.

\section{In vitro fluorescence imaging}

To show the specific targeting ability of our fabricated CMSN@SRT@Anti to macrophages, RAW264.7, $\mathrm{NIH}-3 \mathrm{~T} 3, \mathrm{RAW} 264.7$ pre-treated with $60 \mu \mathrm{g} / \mathrm{mL}$ ox-LDL or NIH-3T3 pre-treated with $60 \mu \mathrm{g} / \mathrm{mL}$ ox-LDL were first inoculated in 6-well plates, respectively $(n=3)$. Then, the cells were then incubated with CMSN@SRT@Anti or CMSN@SRT with a concentration of $16 \mu \mathrm{g} / \mathrm{mL}$ for $2 \mathrm{~h}$ at $37^{\circ} \mathrm{C}$. After fixed with $4 \%$ paraformaldehyde at room temperature for $15 \mathrm{~min}$, the cells were washed twice with PBS and the nuclei were stained with DAPI. The cellular fluorescence imaging were observed under an inverted fluorescence microscope (DMI3000B, Leica, Germany). 


\section{Development Of Animal Model}

The purchased six week-old male ApoE-/-mice (male, GemPharmatech, Nanjing, China), initially fed a normal diet feeding 1 week to adapt to the new environment. Then, mice continued to be fed with the normal diet as a negative control group (low-fat diet, LFD) and a (high-fat diet, HFD) for 12 weeks to establish AS model. Composition of HFD is $40 \%$ Fat and $1.25 \%$ Cholesterol. Kunming male mice (Xuzhou Medical University, Xuzhou, China) were used to study the potential toxicity and metabolism of our prepared nanomaterials. All animal procedures followed the regulations of the Chinese Physiological Society for the Management of Laboratory Animals. The experimental scheme was in line with the ethical requirements of the Animal Care Committee of Xuzhou Medical University (202008A077).

\section{In vivo targeted fluorescence imaging}

In order to select the best imaging time point of nanoparticles in vivo, 5 male AS-model mice (HFD for 16 w) with similar weight and status were used. 4 of them were treated with intraperitoneal injection with CMSN@SRT@Anti (170 mg/kg) respectively. The mice were sacrificed at different time points $(0,1,4,8$, $24 \mathrm{~h}$ ), and the isolated aortas were collected. Then, the arteries were washed with saline three times and visualized using a small animal fluorescence imaging system.

To further compare the specific targeting ability of CMSN@SRT@Anti and CMSN@SRT, the AS-model mice were intraperitoneal injection with PBS, CMSN@SRT@Anti or CMSN@SRT with an amount of 170 $\mathrm{mg} / \mathrm{kg}(\mathrm{n}=3)$, respectively. Four hours later, the isolated aortas were washed with saline and placed in a small animal fluorescence imaging system. The signal intensities of whole arteries were quantified using Tanon Image Gel software (Tanon, Shanghai, China).

In vivo therapy

To evaluate the therapy effect of AS by CMSN@SRT@Anti in vivo, ApoE-/-mice (HFD for 12 w) were divided into five groups $(n=4)$ : the LFD + PBS group (LFD), the HFD + PBS group (Vehicle), the HFD + CMSN@SRT@Anti, the HFD + CMSN@SRT, and the HFD + free SRT1720 group (SRT1720). AS-model mice fed with HFD were intraperitoneal injected with nanomaterials $(170 \mathrm{mg} / \mathrm{kg} / \mathrm{d})$ or SRT1720 $(50 \mathrm{mg} / \mathrm{kg} / \mathrm{d})$ [38] or the same volume of PBS liquid every other day for four weeks. The mice were sacrificed after the treatment. The TC values in blood were determined by the TC determination kit, and the aorta red staining areas after oil red staining were analyzed semi-quantitatively by ImageJ. H\&E was introduced to show the changes of the main organs of mice afterCMSN@SRT@Anti treatment.

The Oil Red $\mathrm{O}$ staining procedure to observe the plaque of lipid deposits was as follows. The aorta was dissected, and the adventitial fat was removed after being opened longitudinally with the luminal surface toward the outside. After fixed in $4 \%$ paraformaldehyde at room temperature for $2 \mathrm{~h}$, the aorta was placed in $0.3 \%$ Oil Red 0 working solution (Solarbio, Beijng, China) at $45^{\circ} \mathrm{C}$ for $1 \mathrm{~h}$ in the dark condition, and then rinsed with $85 \%$ isopropanol for 3 times. The ImageJ quantitatively analyzed the aorta (thoracic aorta, abdominal aorta and aortic arch). 


\section{Statistical analysis}

All the analyses involved in this project were performed by SPSS 22.0 software. Data were expressed as mean \pm standard deviation. Shapiro-Wilk test was used to determine the normality of the data distribution. The multi-group comparisons were made with a one-way ANOVA analysis, followed by Dunnett's post hoc test. The Student's $t$ test was used for comparison between the two groups. According to the test level $a=0.05, P<0.05$ was considered to be statistically significant.

\section{Abbreviations}

AS

Atherosclerosis; CHD:Coronary heart disease; CVD:Cardiovascular disease; HFD:High-Fat diet; LDL:Lowdensity lipoprotein; MSN:Mesoporous silica nanoparticle; NMs:Nanomedicines; Ox-LDL:Oxidized low density lipoprotein; TC:Total cholesterol; TEOS:tetraethoxysilane.

\section{Declarations}

\section{Acknowledgements}

Not applicable.

\section{Authors' contributions}

QW contributed to this work in terms of characterization of nanomaterials, implementation of in vitro and in vivo experiments, and data collection and analysis. YW designed and synthesized this novel nanoparticle, providing the most important premise for the experiment. QW drafted the paper. SL, XS, XS, $Y D, M Z$ and $L C$ analyzed data and supplemented the paper. $\mathrm{JL}$ and $\mathrm{KX}$ designed the experiment and conducted it. The revision of the paper is done by $\mathrm{JL}$. All authors read and approved the final manuscript

\section{Funding}

This work was supported by the National Natural Science Foundation of China (81771904), the Natural Science Foundation of Jiangsu Province for the Excellent Young Scholars (BK20170054), Qing Lan Project, the Peak of Six Talents of Jiangsu Province (WSN-112), Jiangsu Provincial Medical Youth Talent (QNRC2016776), Six one project of Jiangsu Province (LGY2018083).

\section{Availability of data and materials}

All data generated or analysed during this study are included in this published article and its Additional file 1.

\section{Ethics approval and consent to participate}


All animal experiments were conducted according to the Principles of Laboratory Animal Care of the People's Republic of China and the Guidelines for the Care and Use of Laboratory Animals of Xuzhou Medical University, China.

\section{Competing interests}

The authors declare that they have no competing interests.

\section{Author details}

1 School of Medical Imaging, Xuzhou Medical University, Xuzhou 221006,China. 2 Department of Radiology, Affiliated Hospital of Xuzhou Medical University, Xuzhou 221004, China.

\section{References}

1. Gajardo Al, Henríquez F, Llancaqueo M. Big data, social determinants of coronary heart disease and barriers for data access. Eur J Prev Cardiol. 2020. https://doi.org/10.1177/2047487320922366

2. Morin DP, Homoud MK. Prediction and prevention of sudden cardiac death. Card Electrophysiol Clin. 2017; 9(4): 631-8. https://doi.org/10.1016/j.ccep.2017.07.012

3. Zhu Y, Xian X, Wang Z, et al. Research progress on the relationship between atherosclerosis and inflammation. Biomolecules. 2018; 8(3): 80. https://doi.org/10.3390/biom8030080

4. Schaftenaar F, Frodermann V, Kuiper J, et al. Atherosclerosis: the interplay between lipids and immune cells. Curr Opin Lipidol. 2016; 27(3): 209-15.

https://doi.org/10.1097/MOL.0000000000000302

5. Lu H, Daugherty A. Atherosclerosis. Arterioscler Thromb Vasc Biol. 2015; 35(3): 485-491. https: //doi.org/10.1161/ATVBAHA.115.305380

6. Papageorgiou N, Tousoulis D. Oxidized-LDL immunization for the treatment of atherosclerosis: How far are we? Int J Cardiol., 2016; 222: 93-4. https://doi.org/10.1016/j.ijcard.2016.06.334

7. Shaw PX, Hörkkö S, Tsimikas S, et al. Human-derived anti-oxidized LDL autoa- ntibody blocks uptake of oxidized LDL by macrophages and localizes to atherosclerotic lesions in vivo. Arterioscler Thromb Vasc Biol. 2001; 21(8): 1333- 9. https://doi.org/10.1161/hq0801.093587

8. Foss CA, Bedja D, Mease RC, et al. Molecular imaging of inflammation in the ApoE-/-mouse model of atherosclerosis with lodoDPA. Biochem Biophys Res Commun. 2015; 461(1): 70-5. https://doi. org/10.1016/j.bbrc.2015.03.171

9. Chung EJ. Targeting and therapeutic peptides in nanomedicine for atherosclerosis. Exp Biol Med (Maywood). 2016; 241(9): 891-8. https://doi.org/10.1177/1535370216640940

10. Zhang SH, Xu W, Gao P, et al. Construction of dual nanomedicines for the imaging and alleviation of atherosclerosis. Artif Cells Nanomed Biotechnol. 2020; 48(1): 169-79. https://doi.org/10.1080/ 21691401.2019 .1699823 
11. Glinzer A, Ma XP, Prakash J, et al. Targeting elastase for molecular imaging of early atherosclerotic lesions. Arterioscler Thromb Vasc Biol. 2017; 37 (3):525-33. https://doi.org/10.1161/ATVBAHA. 116.308726

12. Sun $X$, Li W, Zhang $X$, et al. In vivo targeting and imaging of atherosclerosis using multifunctional virus-like particles of simian virus 40. Nano Lett. 2016; 16(10): 6164-71. https://doi.org/10.1021/ acs.nanolett.6b02386

13. Prabhudas $M$, Bowdish D, Drickamer $K$, et al. Standardizing scavenger receptor nomenclature. J Immunol. 2014; 192(5): 1997-2006. https://doi.org/10.4049/jimmunol.1490003

14. Zhao L, Varghese Z, Moorhead JF, et al. CD36 and lipid metabolism in the evolution of atherosclerosis. Br Med Bull. 2018; 126(1): 101-12. https://doi.org/10.1093/bmb/ldy006

15. Yao ST, Sang H, Yang NN, et al. Oxidized low density lipoprotein induces macrophage endoplasmic reticulum stress via CD36. Sheng li xue ba : [Acta physiologica Sinica].2010; 62(5): 433-40.

16. Yu LX, Liu XX, Yuan ZH, et al. SRT1720 alleviates ANIT-induced cholestasis in a mouse model. Front Pharmacol. 2017; 8: 256. https://doi.org/10.3389/fphar.2017.00256

17. Stein S, Matter CM. Protective roles of SIRT1 in atherosclerosis. Cell cycle. 2011; 10(4):640-7. https://doi.org/10.4161/cc.10.4.14863

18. Kitada M, Ogura $\mathrm{Y}$, Koya $\mathrm{D}$. The protective role of Sirt1 in vascular tissue: its relationship to vascular aging and atherosclerosis. Aging. 2016; 8(10): 2290-30 https://doi.org/10.18632/aging.101068

19. Chen YX, Zhang M, Cai Y, et al. The Sirt1 activator SRT1720 attenuates angiotensin Il-induced atherosclerosis in apoE-/-mice through inhibiting vascularinflammatory response. Biochem Biophys Res Commun. 2015; 465(4): 732-8. https://doi.org/10.1016/j.bbrc.2015.08.066

20. Lu CL, Li JJ, Xu K, et al. Fabrication of mAb G250-SPIO molecular magnetic resonance imaging nanoprobe for the specific detection of renal cell carcinoma in vitro. PLoS One. 2014; 9(7): e101898. https://doi.org/10.1371/journal.pone.0101898

21. Povrozin Y, Terpetschnig E. Measurement of Fluorescence Quantum Yields on ISS Instrumentation Using Vinci. Technical Note, 2011.

22. Quader S, Kataoka K. Nanomaterial-enabled cancer therapy. Mol Ther. 2017; 25(7): 1501-13. https:// org/10.1016/j.ymthe.2017.04.026

23. Wang X, Li C, Fan N, et al. Amino functionalized chiral mesoporous silica nanoparticles for improved loading and release of poorly water-soluble drug. Asian J Pharm Sci. 2019; 14(4): 405-12. https:// doi.org/10.1016/j.ajps.2018.04.002

24. Tang FQ, Li LL, Chen D. Mesoporous silica nanoparticles: synthesis, biocompatibility and drug delivery. Adv Mater. 2012; 24(12): 1504-34. https://doi.org/10.1002/adma.201104763

25. Vallet-Regi M, Tamanoi F. Overview of studies regarding mesoporous silica nanomaterials and their biomedical application. Enzymes. 2018; 43: 1-10. https://doi.org/10.1016/bs.enz.2018.07.001

26. Ho TA, Cho YB, Kim YS. Synthesis of hybrid reduced graphene oxide decorated with $\mathrm{Ru}(\mathrm{bpy})_{3}\left({ }_{2+}\right)$ doped silica nanoparticles.J Nanosci Nanotechnol. 2016;16(5): 4534-8. https://doi. 
org/10.1166/jnn.2016.11008

27. Tang FQ, Li LL, Chen D. Mesoporous silica nanoparticles: synthesis, biocompatibility and drug delivery. Adv Mater. 2012; 24(12): 1504-34. https://doi.org/10.1002/adma.201104763

28. Dai Y, Zhang A, You J, et al. Fabrication of AS1411 aptamer functionalized Gd203 based molecular magnetic resonance imaging (mMRI) nanoprobe for renal carcinoma cell imaging. RSC Adv. 2015; 5: 77204- Doi: 10.1039/C5RA17211J

29. Guo Y, Wang Z, Shao H, et al. Hydrothermal synthesis of highly fluorescent carbon nanoparticles from sodium citrate and their use for the detection of mercury ions. Carbon. 2013; 52(2):583-9.

30. Seneviratne A, Hulsmans M, Holvoet P, et al. Biomechanical factors and macrophages in plaque stability. Cardiovasc Res. 2013; 99: 284-93. https://doi.org/10.1093/cvr/cvt097

31. Moore KJ, Sheedy FJ, Fisher EA. Macrophages in atherosclerosis: a dynamic balance. Nat Rev Immunol. 2013; 13: 709-21. https://doi.org/10.1038/nri3520

32. Shioi A, Ikari Plaque calcification during atherosclerosis progression and regression. J Atheroscler Thromb. 2018; 25(4): 294-303. https://doi.org/10.5551/jat.RV17020

33. Ding S, Lin N, Sheng XC, et al. Melatonin stabilizes rupture-prone vulnerable plaques via regulating macrophage polarization in a nuclear circadian receptor RORa-dependent manner. J Pineal Res. 2019; 67(2): e12581. https://doi.org/10.1111/jpi.12581

34. Wang X, Chen S, He J, et al. Histone methyltransferases G9a mediated lipidinduced M1 macrophagepolarization through negatively regulating CD36. Metabolism. 2021; 114: 154 https: //doi.org/10.1016/j.metabol.2020.154404

35. Qin M, Wang L, Li F, et al. Oxidized LDL activated eosinophil polarize macrophage phenotype from M2 to M1 through activation of CD36 scavenger receptor. Atherosclerosis. 2017; 263: 82-91. https://doi.org/10.1016/j.atherosclerosis.2017.05.011

36. Voloshyna I, Teboul I, Kasselman LJ, et al. Macrophage lipid accumulation in the presence of immunosuppressive drugs mycophenolate mofetil and cyclosporin A. Inflamm Res. 2019; 68(9): 78799. https://doi.org/10.1007/s00011-019-01262-8

37. Ma ZM, Tian YJ, Wang ZP, et al. Updated roles of SIRT1 in prostate diseases. Zhong hua Nan Ke Xue, 2016, 22(4): 356-360.

38. Ma S, Motevalli SM, Chen JW, et al. Precise theranostic nanomedicines for inhibiting vulnerable atherosclerotic plaque progression through regulation of vascular smooth muscle cell phenotype switching. Theranostics. 2018; 8(13): 3693-706. https://doi.org/10.7150/thno.24364

39. Kulkarni SS, Cantó C. The molecular targets of resveratrol. Biochim Biophys Acta. 2015; 1852(6): 1114-23. https://doi.org/10.1016/j.bbadis.2014.10.005

40. Torchilin VP. Multifunctional, stimuli-sensitive nanoparticulate systems for drug delivery. Nat Rev Drug Discov. 2014; 13(11): 813-27. https://doi.org/10.1038/nrd4333

41. Du CK, Lin XJ, Xu WJ, et al. Sulfhydrated sirtuin-1 increasing its deacetylation activity is an essential epigenetics mechanism of antiatherogenesis by hydrogen sulfide. Antioxid Redox Signal. 2019; 
30(2):184-97. https://doi.org/10.1089/ars.2017.7195

42. Choi HS, Liu W, Misra P, et al. Renal clearance of quantum dots. Nat Biotechnol. 2007; 25: 1165-70. https://doi.org/10.1038/nbt1340

\section{Figures}

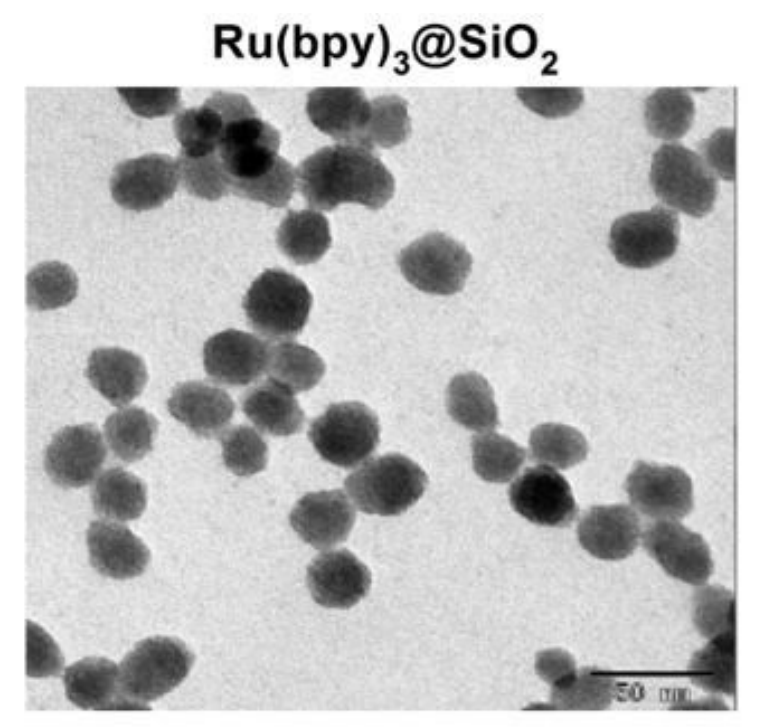

$50 \mathrm{~nm}$



CMSN

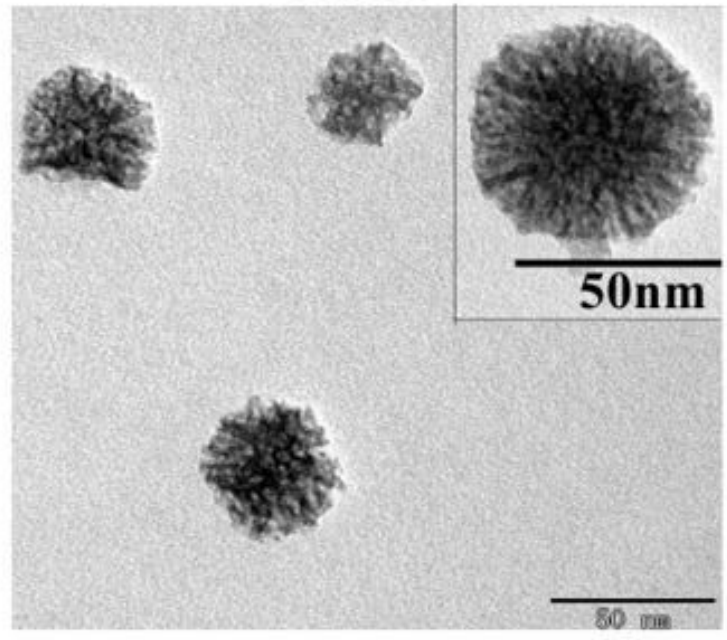

$50 \mathrm{~nm}$

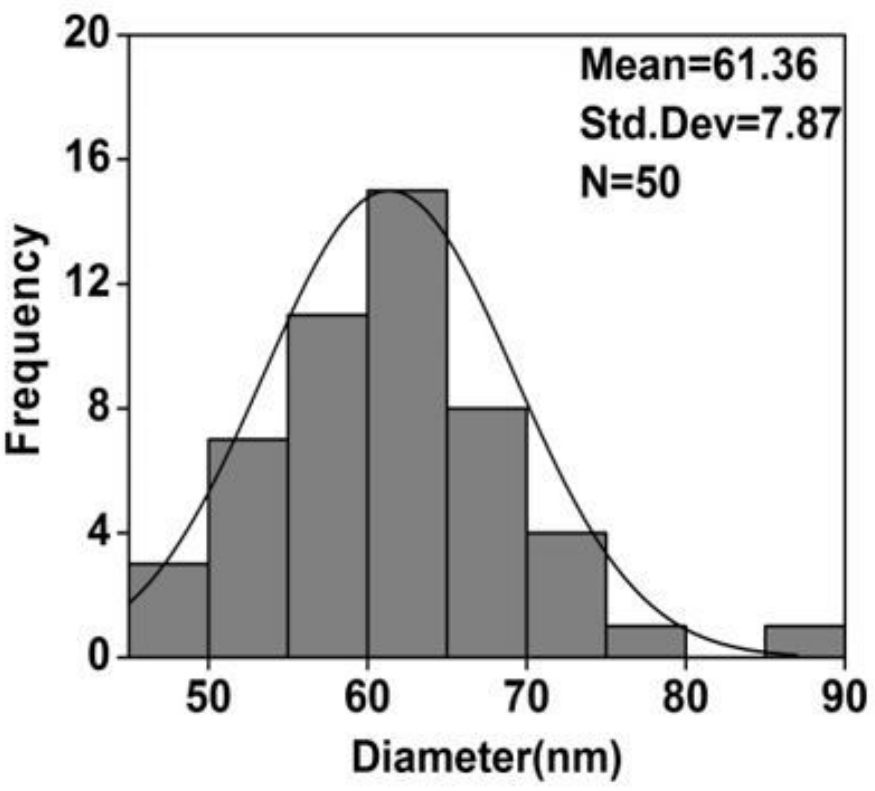

Figure 1

TEM images (Scale bar: $50 \mathrm{~nm}$ ) and size distributions of Ru(bpy)3@SiO2 and CMSN. 

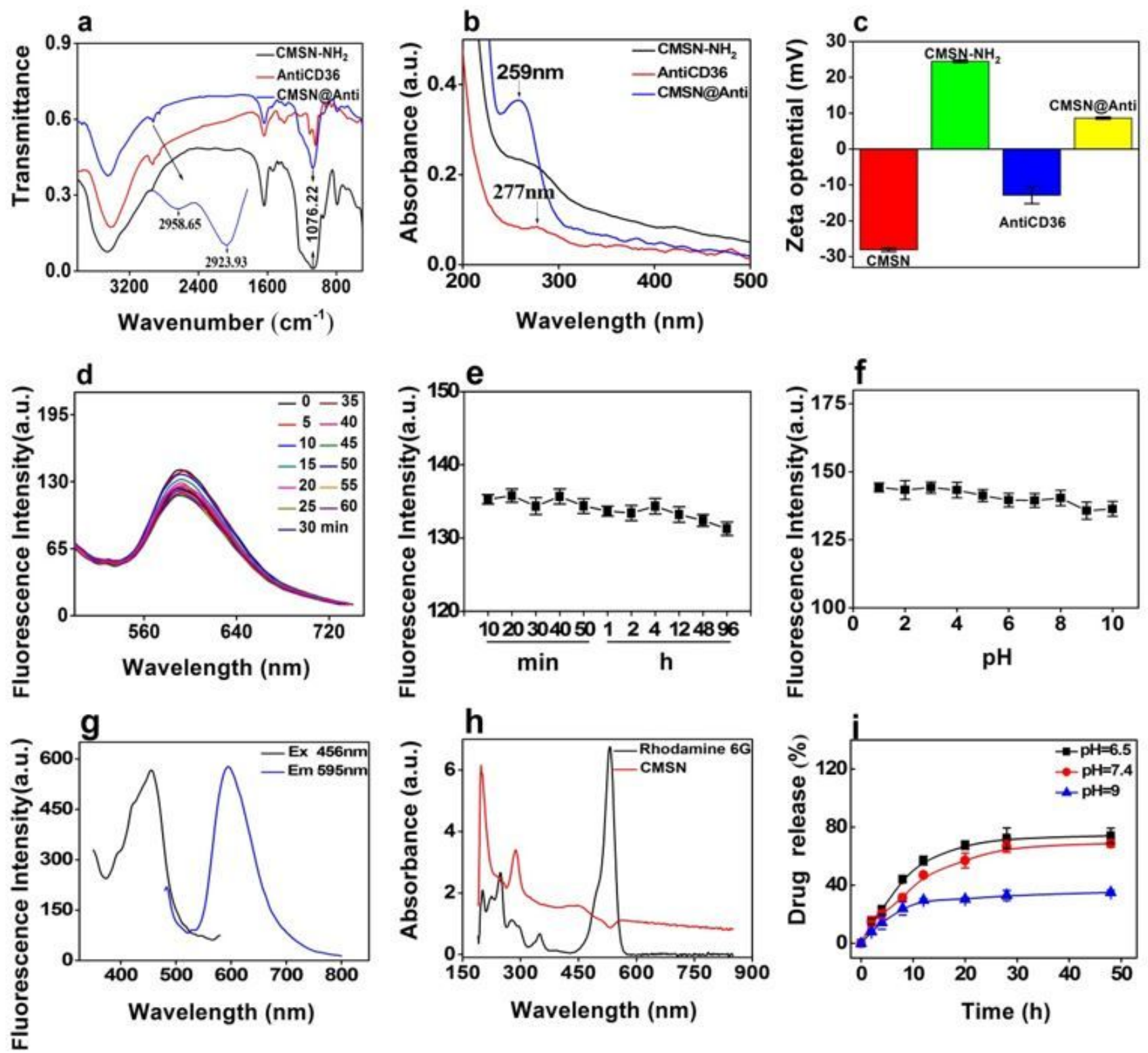

Figure 2

Structure and characteristics of NMs. a-c FTIR, UV-vis and zeta potential of CMSN@Anti. d-f The fluorescence stability of CMSN. $g$ The fluorescence excitation and emission spectra of CMSN. $h$ The UVvis absorption spectra of CMSN and Rhodamine 6G. i The drug release curve of CMSN@SRT@Anti in PBS buffers with different $\mathrm{pH}$ values. 

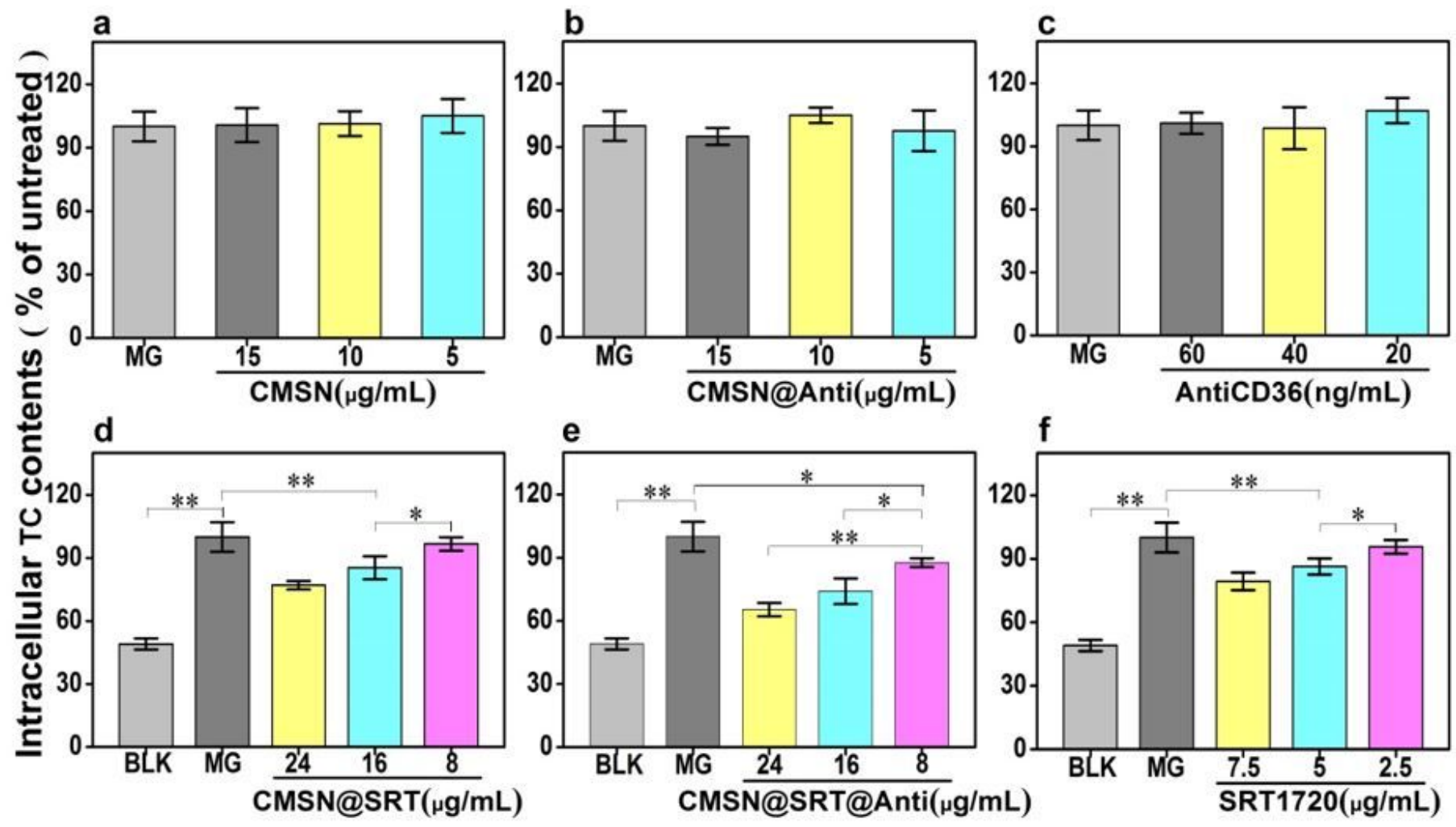

Figure 3

Inhibition effect of different nanomaterials on the foaming process of RAW264.7 cells. a-f The effects of CMSN, CMSN@Anti, AntiCD36, CMSN@SRT, CMSN@SRT@Anti or SRT1720 on TC value of RAW264.7 cells $\left({ }^{*} P<0.05, * * P<0.01\right)$. 

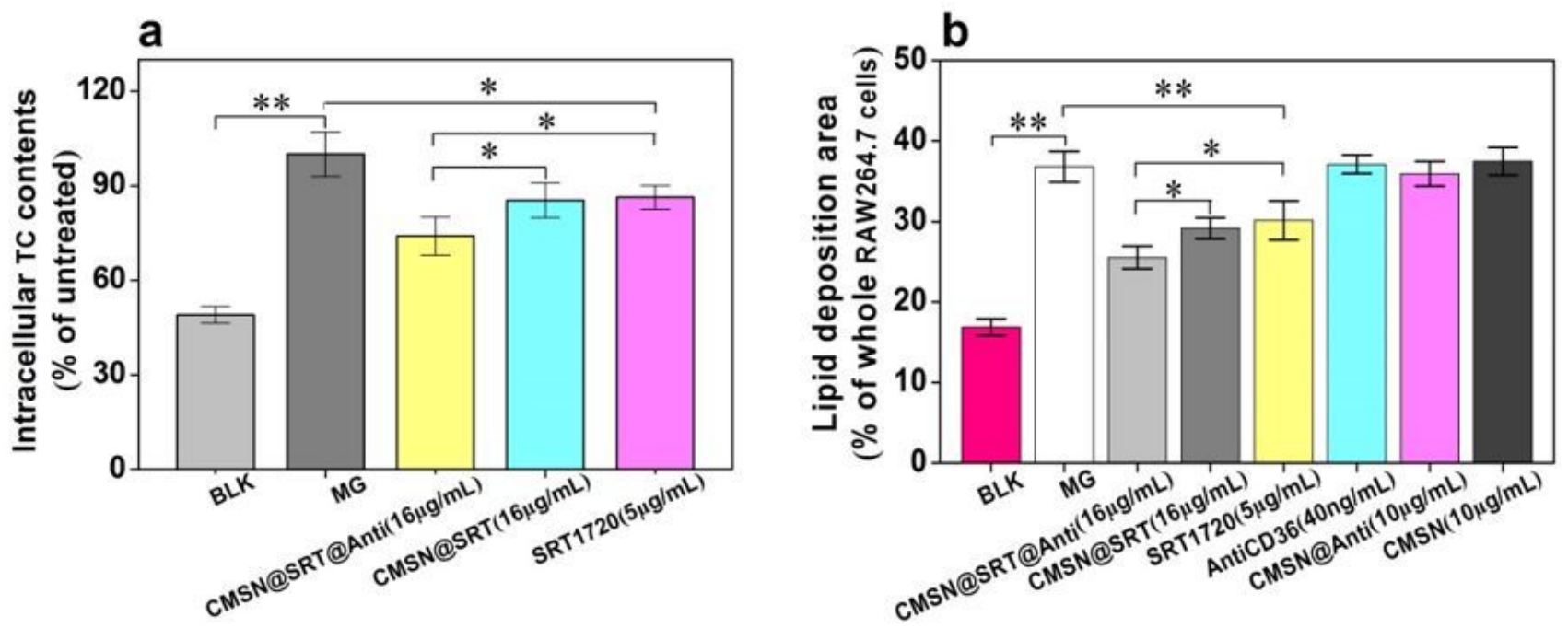

C

RAW264.7 cells


Figure 4

Comparison of RAW264.7cell foaming degree after different intervention. a The effects of different treatments on TC value of RAW264.7 cells ( ${ }^{*} P<0.05$, $\left.{ }^{*} \mathrm{P}<0.01\right)$. b The proportion of Oil Red 0 staining area in RAW264.7 cells after different treatments ( ${ }^{*}<0.05$, $\left.{ }^{*} P<0.01\right)$. c Representative pictures of Oil Red O staining of intracellular lipid droplets (Scale bar: 100 $\mu \mathrm{m}$ ). Note: $16 \mu \mathrm{g}$ of CMSN@SRT@Anti contained about $10 \mu \mathrm{g}$ of CMSN, $40 \mathrm{ng}$ AntiCD36, and released about $5 \mu \mathrm{g}$ SRT1720 within 48 hours. 

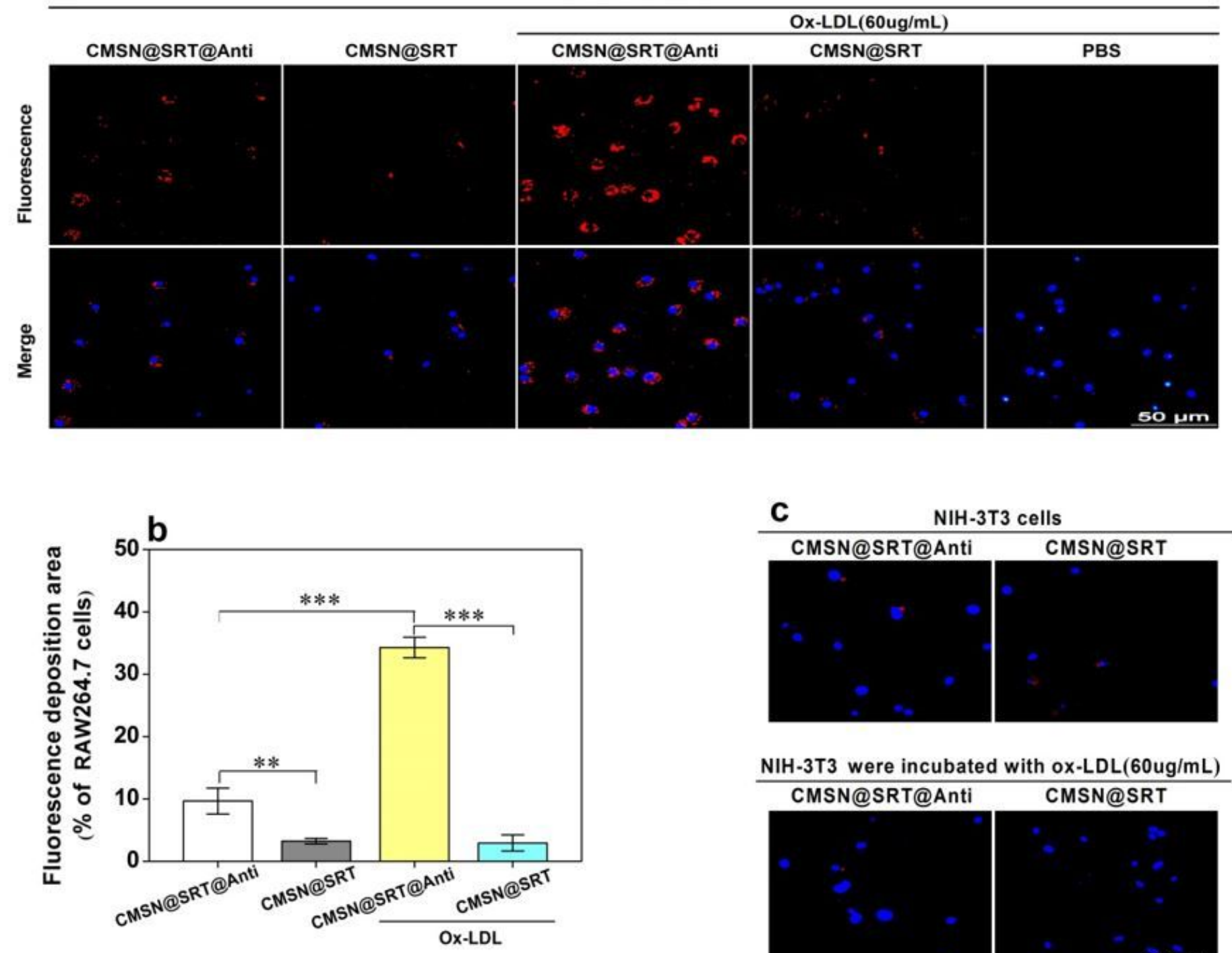

NIH-3T3 were incubated with ox-LDL(60ug/mL) CMSN@SRT@Anti CMSN@SRT
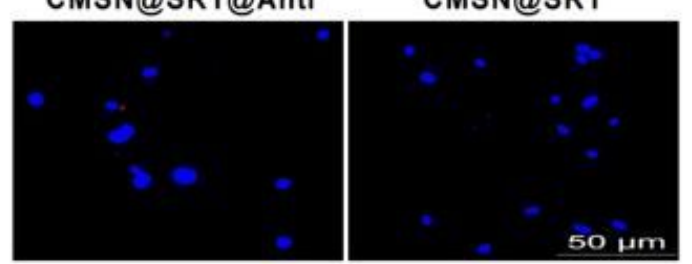

\section{Figure 5}

Targeting property of NMs to cells. a Representative pictures of NMs targeting RAW263.7 cells (Scale bar: $50 \mu \mathrm{m})$. b Quantification of fluorescence area in RAW264.7 cells $(* \star P<0.01, * \star * P<0.001)$. c Targeting property of NMs on NIH-3T3 cells (Scale bar: $50 \mu \mathrm{m}$ ) 


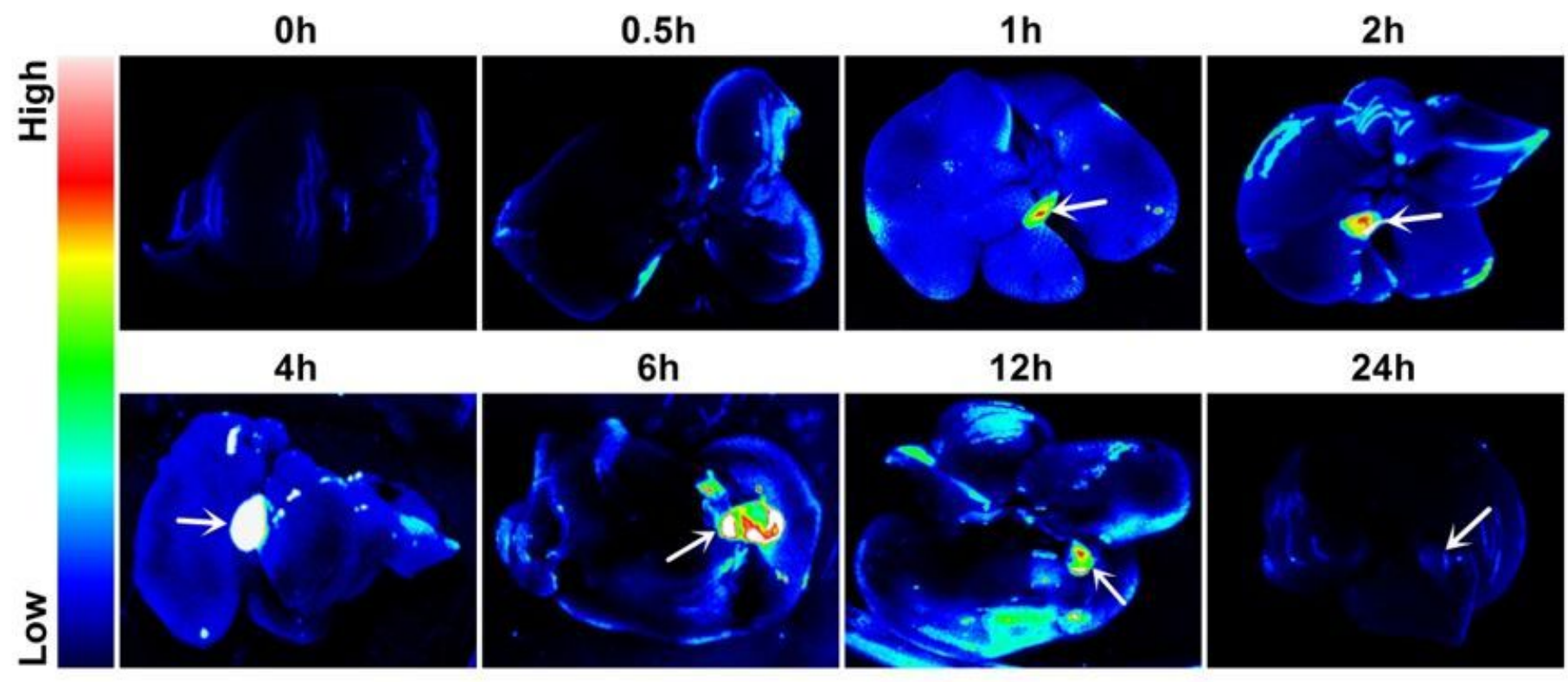

Figure 6

Fluorescence images of liver and cholecyst of Kunming mice at each time point after intraperitoneal injection of CMSN@SRT@Anti (white arrow pointing to cholecyst). 

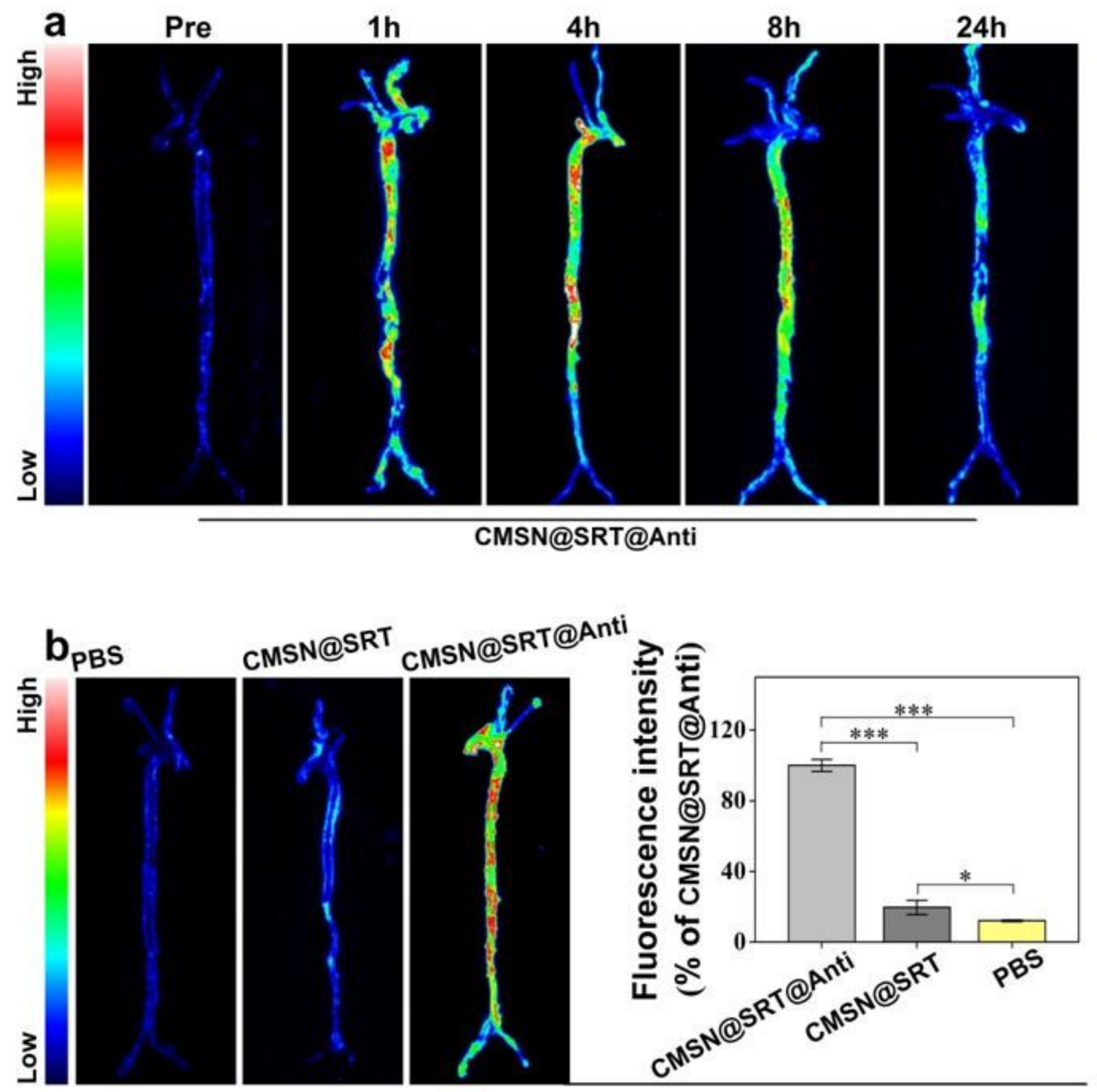

Figure 7

Fluorescence imaging of AS lesions in ApoE-/- mouse aorta. a Fluorescence intensity in AS lesion area of mouse aorta at different time points after intraperitoneal injection of CMSN@SRT@Anti. b Fluorescence imaging of mouse aorta under different treatments and the corresponding histogram of fluorescence intensity $\left({ }^{\star} \mathrm{P}<0.05,{ }^{*} * \mathrm{P}<0.001\right)$. 

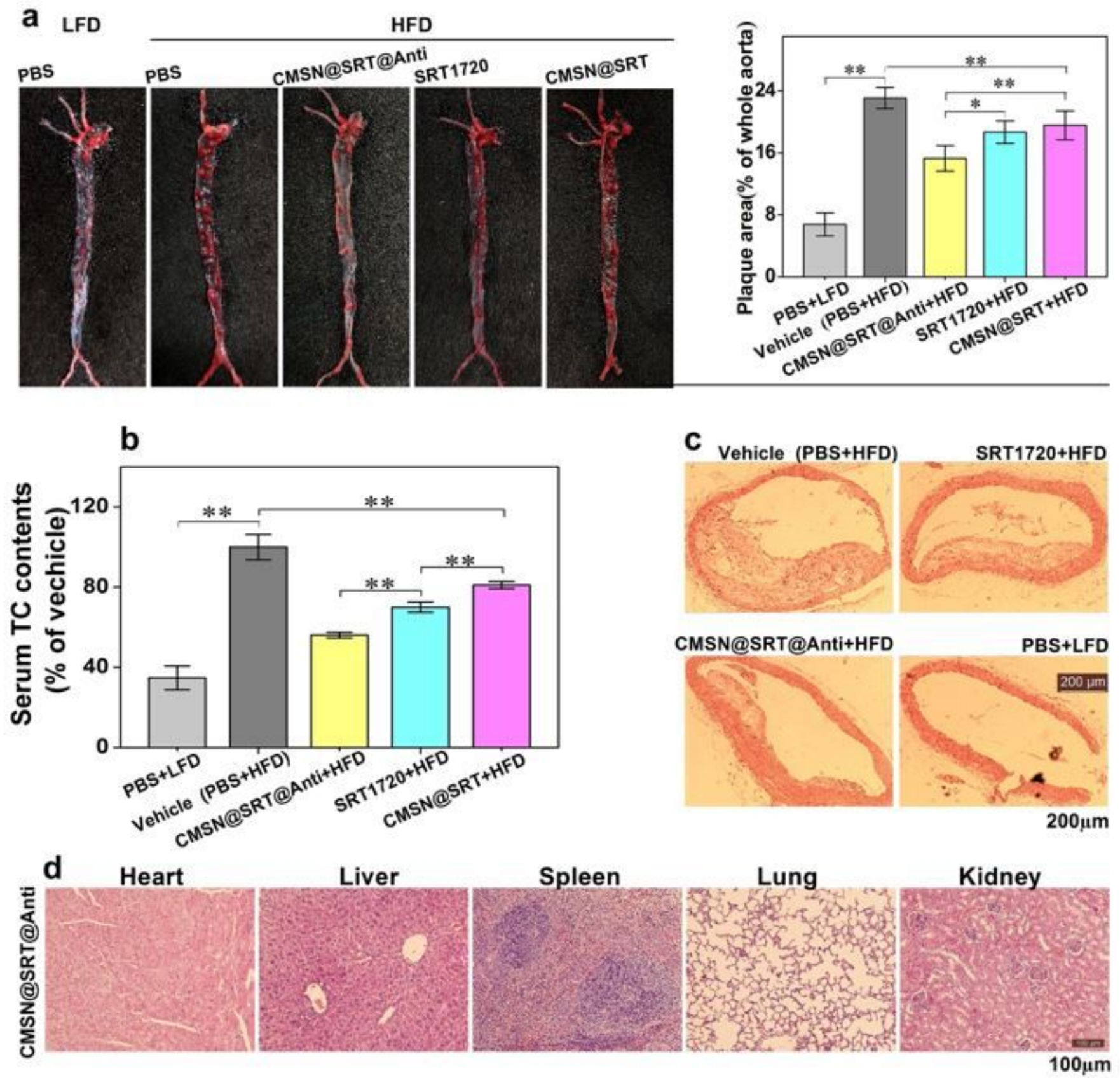

\section{Figure 8}

Inhibition effect of different treatments on the progression of AS in ApoE-/-mice. a Representative pictures of Oil red $O$ staining of mouse aortic plaque and quantification of Oil Red O-stained area $\left({ }^{\star} P<0.05,{ }^{*} \mathrm{P}<0.01\right)$. b Quantitative analysis of $T C$ levels in the mouse serum $\left({ }^{*} \mathrm{P}<0.01\right)$. c Pathological section of aorta in mouse (H\&E, Scale bar: $200 \mu \mathrm{m}$ ). d Pathological section of major organs in mouse (H\&E, Scale bar: $100 \mu \mathrm{m}$ ). Note: About $50 \mathrm{mg}$ SRT1720 can be released in 48 hours by $170 \mathrm{mg}$ CMSN@SRT@anti orCMSN@SRT.

\section{Supplementary Files}


This is a list of supplementary files associated with this preprint. Click to download.

- Graphicalabstract.jpg

- Additionalfile1.docx

- S1.jpg 